\title{
Niveles de ansiedad, depresión y percepción de apoyo social en estudiantes de
} Odontología de la Universidad de Chile

\author{
Levels of Anxiety, Depression and Social Support \\ Perception in Students of Dentistry, University of Chile
}

Araceli Dávila Figueras ${ }^{1}$

Rocío Ruiz Celis ${ }^{2}$

Laura Moncada Arroyo ${ }^{3}$

Iris Gallardo Rayo ${ }^{4}$

\section{Resumen}

Esta investigación tuvo por objetivos describir las variables ansiedad, depresión y apoyo social percibido en estudiantes de Odontología de la Universidad de Chile en dos momentos del semestre académico; y comprobar si con las exigencias curriculares de esta carrera aumentan sus niveles de ansiedad y depresión. Se aplicaron las escalas Hospital Anxiety and Depression Scale -HADS- (Snaith \& Zigmond, 1983) y Duke University Functional Social Support -DUFSS- (Broadhead, De Gruy, Gehlbach \& Kaplan, 1988). El estudio fue transversal y longitudinal, descriptivo, correlacional y con una muestra no probabilística $(n=440)$. Se concluye que los estudiantes se encuentran considerablemente más ansiosos que deprimidos, especialmente los de género femenino y aquellos que cursan tercer y cuarto año.

Palabras clave: ansiedad, depresión, apoyo social, estudiantes universitarios.

1 Psicóloga Universidad de Chile. E-mail: davila.figueras@gmail.com

2 Psicóloga Universidad de Chile. E-mail: rruizcelis@gmail.com

3 Académico Departamento de Psicología. Universidad de Chile. E-mail: lmoncada@uchile.cl

4 Académico Departamento de Psicología. Universidad de Chile. E-mail: igallard@uchile.cl 


\begin{abstract}
This study aimed to describe anxiety, depression and perceived social support variables of University of Chile dental students in two moments in the semester, and also checks the requirements of this curriculum and the increases of the levels of anxiety and depression. The scales Hospital Anxiety and Depression Scale -HADS- (Snaith \& Zigmond, 1983) and Duke University Functional Social Support -DUFSS- were applied. The study was a cross-sectional and longitudinal, descriptive, correlational and with a nonrandom sample $(n=440)$. The findings are that students are significantly more anxious than depressed, especially female, and those who attend to the third and fourth years. The HADS has good reliability, finding a solution of two factors that indicate the original structure of the questionnaire.
\end{abstract}

Key words: anxiety, depression, social support, university students.

\title{
Introducción
}

Diversos autores han mostrado preocupación por la salud mental de los estudiantes universitarios, argumentando que el conjunto de actividades académicas puede influir en su bienestar físico y psicológico, llegando incluso a contribuir a la aparición de cuadros ansiosos o depresivos (Bagés et al., 2008). En nuestro país, si bien no se ha estudiado ampliamente este tema, existe la iniciativa de promover la calidad de vida en el mundo universitario, tal como plantea la Guia para Universidades Saludables y otras Instituciones de Educación Superior (Lange \& Vio, 2006), a la cual se encuentra adscrita la Universidad de Chile.

Según Jerez, Rioseco, Valdivia, Vicente y Vielma (1996), los estudiantes universitarios corresponden a un grupo bien delimitado que tiene como principal actividad estudiar y que, además, comparte una serie de características, como la edad, un nivel intelectual común y una misma escolaridad. Este es además un grupo de individuos que en el futuro ejercerán roles importantes en la sociedad. 
En términos evolutivos, muchos de los estudiantes de pregrado se encuentran en la adolescencia tardía, etapa de transición de la adolescencia a la edad adulta. Este período de moratoria psicosocial que ofrece la sociedad al joven (de acuerdo a la cultura y subcultura), prolonga la adolescencia, permitiéndole al sujeto ubicarse paulatinamente dentro de la sociedad. Es por ello que las exigencias de estudio y perfeccionamiento, en algunos niveles sociales, contribuyen a extender dicho período (Almonte, Correa \& Montt, 2003). Además, el adolescente va definiendo, mediante la adopción de valores, la orientación que mantendrá en su vida en relación a metas, fines y proyectos. En este proceso, los agentes educativos adquieren especial importancia, ya que contribuyen a configurar la forma de pensar y actuar de los estudiantes (Carrasco \& Osses, 2005). De este modo, ellos van adquiriendo su propia personalidad, o comportamiento habitual de cada sujeto, donde interactúan aspectos tanto biológicos como ambientales-sociales para su formación (Pervin, 1998, citado en Reynaldos \& Saavedra, 2006). Entre estos últimos, son la familia y los ambientes académicos los que entregan elementos fundamentales.

Es así como en esta fase de transición a la vida adulta los individuos pueden experimentar mayor estrés, pues se encuentran en un período de formación de la identidad, exploración de la sexualidad e intimidad, independencia de los padres y elección de la carrera, todo lo cual, eventualmente, podría repercutir en su salud mental (Royal College of Psychiatrists, 2003, citado en Kitamura \& Matsudaira, 2006). Según Alonso (1998): "Los niveles de satisfacción, adaptación y rendimiento expresados por un sujeto dependerán de variables como la personalidad, los estilos cognitivos, las expectativas y las tensiones ambientales" (Alonso, 1998, citado en Reynaldos \& Saavedra, 2006).

El paso del colegio a la Universidad constituye un cambio muy importante para los jóvenes, tanto en términos sociales como académicos. El sistema universitario requiere de ellos una mayor autonomía, adecuada distribución del tiempo personal, seguridad en la toma de decisiones y adaptación a la nueva cultura organizacional, lo cual afecta su estilo de vida (Lange \& Vio, 2006). 
Debido a lo señalado anteriormente, existe gran cantidad de investigaciones a nivel internacional que han evaluado la sintomatología tanto ansiosa como depresiva al interior de dicha población (Arrivillaga, Cortés, Goicochea \& Lozano, 2004; Azzam, Chandavarkar \& Mathews, 2007; Bates, Dorian, Greig, Pickard \& Saint, 2000).

Una investigación realizada en el Reino Unido (Bates et al., 2000) midió ansiedad y depresión en estudiantes de $2^{\circ}$ año de Medicina a través de la escala Hospital Anxiety and Depression Scale (HAD). La prevalencia de ansiedad clínicamente significativa fue de 41,2\% (37\% en los hombres y $43,3 \%$ en las mujeres), y la prevalencia de depresión clínicamente significativa fue de $9,5 \%$ ( $9 \%$ en los hombres y $10 \%$ en las mujeres). Estos altos niveles reflejaron la presión para tener éxito, el trabajo arduo, la competencia y las presiones financieras experimentadas por muchos estudiantes hoy en día.

Azzam et al. (2007) evaluaron aproximadamente a 1.050 estudiantes de Medicina de la Universidad de California, la mayoría de edades cercanas a los 25 años, concluyendo que los alumnos de tercer año pueden ser algo más propensos a experimentar síntomas ansiosos y depresivos, con respecto a los de los otros años. En esta muestra, el 5\% de los estudiantes cumplió el criterio para la depresión moderada y el 11,5\% para el trastorno de ansiedad. El género femenino nuevamente aparece asociado a mayores niveles de sintomatología ansiosa y depresiva (Azzam et al., 2007).

Asimismo, diversas investigaciones han estudiado la posible asociación entre depresión y ansiedad en estudiantes universitarios (Carrillo \& Castro, s/f; Baloglu, Karagozoglu \& Masten, 2005). En México (Carrillo et al., s/f) se aplicó el GHQ a 13.342 alumnos de 19,8 años de edad promedio, y a una prevalencia de un $3,8 \%$ de sintomatología ansiosa y un $2,1 \%$ de sintomatología depresiva, se agrega una correlación significativa, directa y moderada $(r=0,651 \mathrm{p}<0,01)$ entre ambas variables. El 65\% de los casos con ansiedad reportó también sintomatología depresiva con manifestaciones de alteraciones del sueño, ingesta, tristeza y melancolía (Carrillo et al., s/f). Por otra parte, un estudio de Turquía que utilizó el BDI y la STAI (Baloglu et al., 2005) hizo la distinción entre rasgo de ansiedad y estado de ansiedad en estudiantes universitarios (edad promedio igual a 20,8 años), 
demostrando que, si bien ambos revelan correlaciones significativas con la depresión, el primero de ellos se relaciona más con esta última que el segundo. Los coeficientes de determinación fueron 0,27 entre depresión y estado de ansiedad, y 0,41 entre depresión y rasgo de ansiedad (Baloglu et al., 2005).

En investigaciones llevadas a cabo con alumnos de Odontología, un estudio longitudinal del Reino Unido (Kamali, Lowry \& NewburyBirch, 2002) revela que la prevalencia de ansiedad patológica fue más alta durante el último año de estudios. En esta investigación se utilizó la escala HAD para medir ansiedad y depresión en estudiantes de Odontología y Medicina, obteniéndose los siguientes resultados: con respecto a la ansiedad patológica, un porcentaje de 47\% ( $2^{\circ}$ año), 67\% ( $5^{\circ}$ año) y 16\% (como dentistas titulados), comparado con un $47 \%$, 26\% y $30 \%$ respectivamente en la carrera de Medicina. Los índices de depresión en los estudiantes de Odontología fueron disminuyendo progresivamente (15\%, 14\% y $2 \%$ ) en contraposición a los de Medicina (4\%, 5\% y 10\%, respectivamente).

En un estudio similar realizado en Chile (Jerez et al., 1996), se concluyó que el 17\% de los estudiantes de Odontología y el 44\% de los estudiantes de Medicina eran casos propensos a presentar un cuadro del área ansioso-depresiva. La presencia de esta sintomatología era más acentuada en aquellos que cursaban primer año, estabilizándose en quinto y disminuyendo considerablemente en el último año. Una de las conclusiones de este estudio es la fuerte asociación entre la desestructuración y la disfunción familiar, y el riesgo de enfermar psíquicamente. En una investigación más reciente (Alvial et al., 2007), efectuada en la mayoría de las facultades de la misma Universidad (632 estudiantes de ambos sexos, con una edad promedio de 21 años), se obtuvo mediante el Inventario de Depresión de Beck y el Inventario de Ansiedad de Beck una prevalencia de un 16,4\% para el síndrome depresivo ( $22 \%$ en las mujeres y $10,6 \%$ en los hombres) y un $23,7 \%$ para el síndrome ansioso (23,8\% en las mujeres y $10,7 \%$ en los hombres), no existiendo diferencias significativas en función de la edad y el nivel de estudios (Alvial et al., 2007).

Diversos teóricos se han interesado por investigar especialmente la salud mental de los estudiantes de carreras como Medicina y 
Odontología, quienes al final de su formación serán directamente responsables de la salud de otras personas (Azzam et al., 2007). Estas carreras se caracterizan por ser altamente demandantes, tanto en términos académicos como psicológicos, lo cual se ve exacerbado cuando además los estudiantes tienen una pobre relación con sus profesores (Bella et al., 2006). Junto con exigir el aprendizaje de una gran cantidad de información en períodos cortos, contemplan dentro de su plan de estudios el trato con pacientes (González, Granadillo, Lara \& Lugo, 2004). Según Bayona et al. (2005), este es un evento de intensa carga emocional que puede ir acompañado por sentimientos de frustración, temor, rabia o desesperación por parte del profesional, lo cual es entendido por otros como Síndrome de Burnout (Maslach, 1982, citado en Acosta, Díaz \& Lartigue, 2001). Entre los factores desencadenantes de estrés en la práctica odontológica se encuentra la rutina, la administración del consultorio, la soledad y los pacientes ansiosos y poco colaboradores, lo que acarrea diversos daños a la salud del profesional, tales como cansancio, irritación, impaciencia, dificultad en la concentración y desánimo (Dossi, Isper, Presta, Saliba C. \& Saliba O., 2006). También constituyen estresores las relaciones tensas entre los compañeros de trabajo y con el equipo auxiliar, los atrasos de los pacientes y la presión social para trabajar rápido y sin errores, situaciones que, si se prolongan en el tiempo, pueden aumentar la ansiedad y generar patologías como el Síndrome de Burnout (Acosta et al., 2001; Dossi et al., 2006).

Cabe recordar que la aparición de síntomas ansiosos o depresivos se encuentra mediada por factores tanto personales como psicosociales. Dentro de estos últimos, el apoyo social ha sido ampliamente estudiado debido a su influencia en el bienestar psicológico de las personas (Barrón \& Sánchez, 2001; Bovier, Chamot \& Perneger, 2004; Cornejo \& Lucero, 2005; González \& Landero, 2006). Según Bagés et al. (2008): “El apoyo social puede darse a través de todos aquellos que rodean a la persona y los recursos que le brindan, lo cual proporciona un soporte emocional tangible” (p. 740). La dimensión subjetiva de este fenómeno, como percepción y significado otorgado por el individuo a la presencia de dichos elementos, constituye un factor protector de la salud mental que ha sido ampliamente 
estudiado (Bagés et al., 2008). Esto cobra mayor relevancia a la luz de la evidencia encontrada acerca del desgaste emocional propio de la labor odontológica (Dossi et al., 2006).

A partir de los antecedentes anteriores, surgen las siguientes interrogantes: ¿Cómo se manifiesta la ansiedad y la depresión en dos momentos del semestre diferenciados por las exigencias académicas, en los estudiantes de $1^{\circ}$ a $5^{\circ}$ año de Odontología de la Universidad de Chile; y cómo se relacionan estas variables con el género y el apoyo social percibido?

La presente investigación tiene dos objetivos generales. El primero de ellos corresponde a: Describir las variables ansiedad, depresión y apoyo social percibido en estudiantes universitarios de la carrera de Odontología de la Universidad de Chile en dos momentos del semestre, diferenciados por el número de exigencias propias del currículum académico. El segundo objetivo es: Comprobar si los niveles de ansiedad y depresión de los estudiantes de Odontología de la Universidad de Chile aumentan cuando estos se ven expuestos a mayores exigencias curriculares.

Interesa también comparar las variables ansiedad y depresión por curso y género. Por otro lado, se medirá la percepción de apoyo social funcional, como recurso que puede mitigar los síntomas señalados. Se establecerán correlaciones entre las tres variables y, adicionalmente, se llevará a cabo el cálculo de la confiabilidad y validez de la Hospital Anxiety and Depression Scale (HADS), escala que se utilizará para evaluar ansiedad y depresión.

Las principales hipótesis propuestas son: La medición realizada en período de evaluación de conocimientos (Muestra POST) arrojará puntajes significativamente superiores en la escala de ansiedad y en la escala de depresión a los puntajes obtenidos en la medición realizada a inicios del semestre (Muestra PRE) en estas escalas, en los estudiantes de Odontología de la Universidad de Chile.

En relación con la variable género, la hipótesis planteada es: El género femenino presentará un mayor nivel de sintomatología ansiosa y depresiva respecto del género masculino en los estudiantes de Odontología de la Universidad de Chile, en ambos momentos de evaluación. 
Por último, respecto a la variable Apoyo Social, se propone la hipótesis: La percepción de apoyo social no varía significativamente en los dos momentos del semestre en que los estudiantes de Odontología de la Universidad de Chile se ven expuestos a distinta presión académica.

El interés por este tema radica en la escasez de investigaciones que abordan esta problemática y en las características de la carrera de Odontología, particularmente al interior de esta Universidad. Entre estas se encuentra una alta carga académica, tanto en la etapa básica como clínica, junto con modos de evaluación que ponen énfasis en la parte práctica, dando mayor importancia al área psicomotora y afectiva (Universidad de Chile, 2006). La presente investigación es entonces relevante en términos institucionales, para el diseño de estrategias en el marco del Programa de Universidades Saludables, al cual pertenece la Universidad de Chile. Cabe señalar que esta investigación nace como un proyecto interfacultades, surgiendo al interior de la misma Facultad de Odontología la necesidad de evaluar la ansiedad y depresión dentro de este contexto.

\section{Método}

\section{Participantes}

La población está conformada por todos los alumnos y alumnas de la carrera de Odontología de la Universidad de Chile. Las unidades de análisis corresponden a los estudiantes de $1^{\circ}, 2^{\circ}, 3^{\circ}, 4^{\circ}$ y $5^{\circ}$ año de esta carrera. El criterio de inclusión de la muestra fue estar cursando alguna de las asignaturas de estos cursos, durante el segundo semestre del año 2009.

El muestreo es de tipo no probabilístico, accidental o errático. El número de la muestra se obtuvo a partir de los estudiantes que quisieran participar voluntariamente de esta investigación al momento de asistir a los cursos, y fue igual a 440 sujetos, correspondientes al 76,16\% del total de alumnos matriculados de $1^{\circ}$ a $5^{\circ}$ año (579 sujetos). Cabe destacar que en cada uno de los cursos se contó con más del $60 \%$ de los alumnos para incluir 
la muestra, por lo cual se puede afirmar que la muestra es representativa del colectivo. Las edades de los participantes fluctuaron entre 18 y 31 años de edad, con una edad promedio de 21,64 años ( $\mathrm{DE}=2,70)$.

\section{Instrumentos}

Para recolectar los datos se administró una batería de instrumentos que contenía los cuestionarios HADS y Duke University Functional Social Support (Versión española, Bellón et al., 1996), los cuales fueron aplicados en dos oportunidades durante el segundo semestre académico del año 2009.

La HADS corresponde a un instrumento autoaplicado, constituido por 14 ítems, que posee una subescala de ansiedad de 7 ítems (HAD-A) y una subescala de depresión de 7 ítems (HAD-D). Esta última se centra en evaluar la pérdida de respuesta al placer (anhedonia) (Snaith \& Zigmond, 1983; Snaith, 1992). Se utilizará la interpretación recomendada por los autores de la HADS, quienes, a partir de los puntajes brutos en ambas escalas, establecen las siguientes categorías (Snaith \& Zigmond, 1983, citado en Bascarán et al., 2008).

$0-7=$ Normalidad

8-10=Caso Probable

\section{1-21=Caso de Ansiedad o de Depresión}

Tanto para la subescala de ansiedad como para la subescala de depresión, la puntuación se obtiene sumando los puntajes obtenidos por los sujetos en cada uno de los ítems que las conforman.

Por otro lado, el cuestionario Duke University Functional Social Support (DUFSS) fue diseñado por Broadhead, De Gruy, Gehlbach y Kaplan (1988) con el propósito de medir de manera breve, confiable y válida el Apoyo Social Funcional, a raíz de la evidencia encontrada acerca de 
que la calidad, más que la cantidad de apoyo, ejerce un efecto beneficioso sobre la salud. Este instrumento consta de 8 ítems, distribuidos en dos dimensiones: Apoyo Confidencial (posibilidad de contar con personas para comunicarse) y Apoyo Afectivo (demostraciones de amor, cariño y empatía). Sin embargo, dos validaciones españolas han optado por la versión de 11 ítems, la misma que es utilizada en el presente estudio, cuyo puntaje de corte sugerido es 32 . De acuerdo a ello, los sujetos con un puntaje menor a 32 presentan un apoyo social bajo y quienes obtengan un puntaje superior a 32 presentarán un apoyo social normal (Bellón et al., 1996). Los resultados del cuestionario son un reflejo del apoyo social percibido, por lo tanto, a menor puntuación, menor percepción de apoyo social.

Para ambos instrumentos, el sujeto contesta a cada ítem utilizando una escala tipo Likert, en la cual él mismo evalúa sus síntomas o su percepción de apoyo social.

\section{Procedimiento}

La primera medición se llevó a cabo durante la última semana del mes de julio y la primera semana de agosto de 2009, correspondientes al inicio del segundo semestre, período sin mayores exigencias académicas para los estudiantes (Muestra PRE). La segunda medición se realizó durante las dos primeras semanas de octubre del mismo año, momento en el cual los alumnos se encontraban en pleno período de evaluación de conocimientos (Muestra POST).

En ambas ocasiones se ingresó a las salas de clases, laboratorios y clínicas de la Facultad de Odontología de la Universidad de Chile, contando previamente con la aprobación de los docentes a cargo de cada curso. Se les hizo entrega a los alumnos de ambos cuestionarios, acompañados de un Consentimiento Informado, el cual expresaba aspectos relacionados con la confidencialidad del estudio y el carácter voluntario de su participación. 


\section{Análisis de datos}

Se utilizó la versión 15.0 del programa SPSS, a través de estadísticos descriptivos, coeficientes de correlación y pruebas no paramétricas. El nivel de significación utilizado para la interpretación de los resultados es $\alpha=0,05$. Cabe señalar que, para efectos de las correlaciones, se llevaron a cabo los cálculos a partir de los puntajes brutos obtenidos por los sujetos de la muestra, a diferencia de los demás resultados, en los cuales se ha optado por el uso de las categorías de ambos instrumentos.

\section{Criterios de Confiabilidad y Validez}

A continuación se expondrá el análisis de confiabilidad y validez del instrumento utilizado para evaluar ansiedad y depresión (HADS). Para aportar a la confiabilidad y validez de constructo del HADS, se ha optado por considerar los resultados obtenidos en el segundo momento de medición ( $\mathrm{n}=344)$, pues en este los sujetos presentaban una configuración más nítida de sus estados anímicos, ya que se encontraban en período de pruebas (Muestra POST). Sin embargo, en las Tablas 1 y 2 se presentan algunos estadísticos descriptivos correspondientes a ambas muestras (muestra PRE y muestra POST), con el fin de contemplar las diferencias por año cursado, género y momento del semestre, en relación a las tres variables evaluadas: Ansiedad, Depresión y Apoyo Social.

Respecto a las características de cada subescala de la HADS, el promedio de la subescala de ansiedad es de 10,08 (D. E. = 4,07), mientras que la media de la subescala de depresión es de 6,01 (D. E. = 3, 38). La subescala de ansiedad presentó una buena confiabilidad, con un Alfa de Cronbach basada en los elementos tipificados de 0,83. La subescala de depresión presentó un Alfa de Cronbach basada en los elementos tipificados un poco menor que el arrojado para la subescala de ansiedad, correspondiente a 0,74 . Al examinar la variación de la confiabilidad de ambas subescalas si algunos de sus ítems son eliminados, se observa que esta no aumenta, por lo cual no es necesario extraer ninguno de ellos, debido a que miden consistentemente. 
Tabla 1

Estadísticos según año cursado y género de las variables PRE

\begin{tabular}{|c|c|c|c|c|}
\hline Curso & Estadístico & $\begin{array}{l}\text { Ansiedad } \\
\text { PRE }\end{array}$ & $\begin{array}{c}\text { Depresión } \\
\text { PRE }\end{array}$ & $\begin{array}{c}\text { Apoyo Social } \\
\text { PRE }\end{array}$ \\
\hline \multirow[t]{3}{*}{ Primer ańo } & $\mathrm{N}$ válidos & 80 & 80 & 80 \\
\hline & Media & 9,66 & 5,79 & 45,23 \\
\hline & $\begin{array}{l}\text { Desviación } \\
\text { estándar }\end{array}$ & 3,44 & 3,15 & 6,41 \\
\hline \multirow[t]{3}{*}{ Segundo año } & $\mathrm{N}$ válidos & 43 & 43 & 43 \\
\hline & Media & 7,79 & 4,67 & 46,30 \\
\hline & $\begin{array}{c}\text { Desviación } \\
\text { estándar }\end{array}$ & 3,07 & 3,49 & 8,46 \\
\hline \multirow[t]{3}{*}{ Tercer año } & $\mathrm{N}$ válidos & 59 & 59 & 59 \\
\hline & Media & 9,80 & 6,39 & 44,46 \\
\hline & $\begin{array}{l}\text { Desviación } \\
\text { estándar }\end{array}$ & 3,65 & 3,14 & 7,65 \\
\hline \multirow[t]{3}{*}{ Cuarto año } & $\mathrm{N}$ válidos & 62 & 62 & 62 \\
\hline & Media & 10,79 & 6,52 & 42,95 \\
\hline & $\begin{array}{c}\text { Desviación } \\
\text { estándar }\end{array}$ & 3,66 & 2,91 & 6,38 \\
\hline \multirow[t]{3}{*}{ Quinto año } & $\mathrm{N}$ válidos & 49 & 49 & 49 \\
\hline & Media & 9,12 & 4,98 & 44,78 \\
\hline & $\begin{array}{l}\text { Desviación } \\
\text { estándar }\end{array}$ & 4,02 & 3,24 & 7,97 \\
\hline Género & Estadístico & $\begin{array}{c}\text { Ansiedad } \\
\text { PRE }\end{array}$ & $\begin{array}{c}\text { Depresión } \\
\text { PRE }\end{array}$ & $\begin{array}{c}\text { Apoyo Social } \\
\text { PRE }\end{array}$ \\
\hline \multirow[t]{3}{*}{ Masculino } & $\mathrm{N}$ válidos & 118 & 118 & 118 \\
\hline & Media & 8,89 & 5,22 & 43,67 \\
\hline & $\begin{array}{l}\text { Desviación } \\
\text { estándar }\end{array}$ & 3,36 & 3,07 & 7,64 \\
\hline \multirow[t]{3}{*}{ Femenino } & $\mathrm{N}$ válidos & 175 & 175 & 175 \\
\hline & Media & 10,02 & 6,13 & 45,35 \\
\hline & $\begin{array}{l}\text { Desviación } \\
\text { estándar }\end{array}$ & 3,81 & 3,27 & 6,97 \\
\hline
\end{tabular}


Tabla 2

Estadísticos según año cursado y género de las variables POST

\begin{tabular}{|c|c|c|c|c|}
\hline Curso & Estadístico & $\begin{array}{l}\text { Ansiedad } \\
\text { POST }\end{array}$ & $\begin{array}{l}\text { Depresión } \\
\text { POST }\end{array}$ & $\begin{array}{c}\text { Apoyo Social } \\
\text { POST }\end{array}$ \\
\hline \multirow[t]{3}{*}{ Primer ańo } & $\mathrm{N}$ válidos & 88 & 88 & 88 \\
\hline & Media & 8,84 & 5,53 & 45,50 \\
\hline & $\begin{array}{l}\text { Desviación } \\
\text { estándar }\end{array}$ & 4,06 & 3,23 & 6,82 \\
\hline \multirow[t]{3}{*}{ Segundo año } & $\mathrm{N}$ válidos & 40 & 40 & 40 \\
\hline & Media & 8,65 & 5,30 & 45,93 \\
\hline & $\begin{array}{c}\text { Desviación } \\
\text { estándar }\end{array}$ & 2,81 & 3,37 & 7,64 \\
\hline \multirow[t]{3}{*}{ Tercer ańo } & $\mathrm{N}$ válidos & 76 & 76 & 73 \\
\hline & Media & 10,58 & 6,75 & 45,70 \\
\hline & $\begin{array}{l}\text { Desviación } \\
\text { estándar }\end{array}$ & 4,34 & 3,57 & 7,45 \\
\hline \multirow[t]{3}{*}{ Cuarto ańo } & $\mathrm{N}$ válidos & 90 & 90 & 89 \\
\hline & Media & 11,67 & 6,74 & 43,64 \\
\hline & $\begin{array}{l}\text { Desviación } \\
\text { estándar }\end{array}$ & 3,68 & 3,01 & 6,57 \\
\hline \multirow[t]{3}{*}{ Quinto año } & $\mathrm{N}$ válidos & 50 & 50 & 49 \\
\hline & Media & 9,76 & 4,98 & 44,82 \\
\hline & $\begin{array}{l}\text { Desviación } \\
\text { estándar }\end{array}$ & 4,17 & 3,59 & 7,73 \\
\hline Género & Estadístico & $\begin{array}{l}\text { Ansiedad } \\
\text { POST }\end{array}$ & $\begin{array}{c}\text { Depresión } \\
\text { POST }\end{array}$ & $\begin{array}{c}\text { Apoyo Social } \\
\text { POST }\end{array}$ \\
\hline \multirow[t]{3}{*}{ Masculino } & $\mathrm{N}$ válidos & 141 & 141 & 139 \\
\hline & Media & 8,80 & 5,36 & 45,25 \\
\hline & $\begin{array}{l}\text { Desviación } \\
\text { estándar }\end{array}$ & 3,91 & 3,39 & 6,88 \\
\hline \multirow[t]{3}{*}{ Femenino } & $\mathrm{N}$ válidos & 203 & 203 & 200 \\
\hline & Media & 10,96 & 6,46 & 44,84 \\
\hline & $\begin{array}{c}\text { Desviación } \\
\text { estándar }\end{array}$ & 3,95 & 3,31 & 7,33 \\
\hline
\end{tabular}


Para comenzar el análisis de validez de constructo se calculó la medida de adecuación muestral de Kaiser-Meyer-Olkin, obteniéndose un valor de 0,90 , lo cual justifica la realización de un análisis factorial exploratorio. Este análisis se realizó a través del método de extracción de componentes principales, con rotación varimax. Se encontró evidencia para una solución de 2 factores, los cuales explican el 46,79\% de la varianza total: Factor I (24,12\%) y Factor II (22,67\%). El Factor I corresponde a la subescala de ansiedad y el Factor II a la subescala de depresión, por lo tanto, la estructura teórica del cuestionario se confirma (Tabla 3). La presencia de dos factores reproduce los hallazgos de la escala original propuesta por Snaith \& Zigmond (1983).

\section{Tabla 3}

Análisis de validez de constructo: Matriz de componentes rotados

Escala de Ansiedad

Escala de Depresión

\begin{tabular}{|c|c|c|c|c|c|}
\hline & \multicolumn{2}{|c|}{ Componente } & & \multicolumn{2}{|c|}{ Componente } \\
\hline & Factor I & Factor II & & Factor I & Factor II \\
\hline Ítem 1 & 0,630 & 0,427 & Ítem 1 & 0,329 & 0,630 \\
\hline Ítem 2 & 0,567 & 0,458 & Ítem 2 & 0,062 & 0,749 \\
\hline Ítem 3 & 0,575 & 0,387 & Ítem 3 & 0,248 & 0,638 \\
\hline Ítem 4 & 0,689 & 0,157 & Ítem 4 & 0,413 & 0,373 \\
\hline Ítem 5 & 0,690 & 0,074 & Ítem 5 & 0,124 & 0,429 \\
\hline Ítem 6 & 0,756 & $-0,025$ & Ítem 6 & 0,019 & 0,720 \\
\hline Ítem 7 & 0,601 & 0,457 & Ítem 7 & 0,291 & 0,431 \\
\hline
\end{tabular}




\section{Resultados}

La medición del nivel de ansiedad reportado por los alumnos en la primera oportunidad fue de $37,5 \%$, mientras que en la segunda oportunidad este correspondió al 45,9\%. Por otra parte, el nivel de depresión informado en la primera evaluación fue de 7,8\%, mientras que en la segunda fue de $9,9 \%$. Se puede observar un incremento en el porcentaje de casos en ambas variables, como se esperaba.

Para comprobar si los niveles de ansiedad y depresión de los estudiantes de Odontología de la Universidad de Chile aumentan cuando estos se ven expuestos a mayores exigencias curriculares, se utilizó la Prueba de rangos señalados de Wilcoxon (Tabla 4), con el fin de realizar comparaciones entre la primera y la segunda medición en estas variables. A partir de este cálculo se puede afirmar que existen diferencias significativas para la ansiedad, sin embargo, no se aprecian diferencias significativas para la variable depresión. En el segundo momento de evaluación los niveles de ansiedad aumentaron, no ocurriendo lo mismo en el caso de la depresión. Con respecto a la variación del apoyo social según las exigencias curriculares, se infiere que existen diferencias significativas en el apoyo social percibido entre la primera y la segunda evaluación.

\section{Tabla 4}

\section{Comparación antes (PRE)- después (POST) de las variables evaluadas}

Prueba de Rangos Señalados de Wilcoxon

\begin{tabular}{ccc}
\hline & $\mathrm{p}$ & $\mathrm{z}$ \\
\hline Ansiedad PRE-Ansiedad POST & $-0,001$ & $-3,205$ \\
Depresión PRE-Depresión POST & 0,664 & $-0,435$ \\
Apoyo Social PRE-Apoyo Social POST & 0,008 & $-2,673$ \\
\hline
\end{tabular}

Por otra parte, los cursos que destacan por su alta presencia de sintomatología ansiosa en ambas oportunidades son $3^{\circ}$ y $4^{\circ}$ (Tablas 5 y 6), no existiendo una tendencia clara en cuanto a la variable depresión. 


\section{Tabla 5}

Frecuencias y Porcentajes de Ansiedad PRE, por año cursado

\begin{tabular}{llllll} 
& \multicolumn{5}{c}{ Año cursado de la carrera } \\
\cline { 2 - 6 } Categorías & $1^{\circ}$ & $2^{\circ}$ & $3^{\circ}$ & $4^{\circ}$ & $5^{\circ}$
\end{tabular}

$\begin{array}{llllllllll}\mathrm{Fr} & \% & \mathrm{Fr} & \% & \mathrm{Fr} & \% & \mathrm{Fr} & \% & \mathrm{Fr} & \%\end{array}$

$\begin{array}{lllllllllll}\text { Normalidad } & 21 & 26,3 & 24 & 55,8 & 20 & 33,9 & 16 & 25,8 & 17 & 34,7\end{array}$

$\begin{array}{lllllllllll}\text { Caso } & 31 & 38,8 & 10 & 23,3 & 15 & 25,4 & 12 & 19,4 & 17 & 34,7\end{array}$

$\begin{array}{lllllllllll}\text { Caso de } & 28 & 35,0 & 9 & 20,9 & 24 & 40,7 & 34 & 54,8 & 15 & 30,6\end{array}$

$\begin{array}{lllllllllll}\text { Total } & 80 & 100,0 & 43 & 100,0 & 59 & 100,0 & 62 & 100,0 & 49 & 100,0\end{array}$

Fr= Frecuencia de casos \%=Porcentaje de casos

Tabla 6

Frecuencias y Porcentajes de Ansiedad POST, por año cursado

\begin{tabular}{cccccccccccc} 
& \multicolumn{8}{c}{ Ańo cursado de la carrera } \\
\cline { 2 - 9 } Categorías & \multicolumn{1}{c}{$1^{\circ}$} & & $2^{\circ}$ & & $3^{\circ}$ & & $4^{\circ}$ & & $5^{\circ}$ \\
& & Fr & $\%$ & Fr & $\%$ & Fr & $\%$ & Fr & $\%$ & Fr & $\%$
\end{tabular}

$\begin{array}{lllllllllll}\text { Normalidad } & 33 & 37,5 & 14 & 35,0 & 20 & 26,3 & 13 & 14,4 & 14 & 28,0\end{array}$

$\begin{array}{lllllllllll}\text { Caso } & 26 & 29,5 & 16 & 40,0 & 14 & 18,4 & 22 & 24,4 & 14 & 28,0\end{array}$

$\begin{array}{lllllllllll}\text { Caso de } & 29 & 33,0 & 10 & 25,0 & 42 & 55,3 & 55 & 61,1 & 22 & 44,0\end{array}$

$\begin{array}{lllllllllll}\text { Total } & 88 & 100,0 & 40 & 100,0 & 76 & 100 & 90 & 100,0 & 50 & 100,0\end{array}$

$\mathrm{Fr}=$ Frecuencia de casos $\%=$ Porcentaje de casos 
Se observa que existen diferencias entre el género femenino y el género masculino en el nivel de sintomatología ansiosa y depresiva (Tablas 7 y 8). Sin embargo, al aplicar la Prueba U de Mann-Whitney, se puede concluir que estas diferencias son significativas solo en el segundo momento de evaluación (Tabla 9).

Tabla 7

Frecuencias y Porcentajes de Ansiedad y Depresión por Género

\begin{tabular}{ccccccc}
\hline Género & \multicolumn{2}{c}{ Ansiedad PRE } & \multicolumn{2}{c}{ Depresión PRE } & \multicolumn{2}{c}{ Total PRE } \\
\hline & Fr & $\%$ & Fr & $\%$ & Fr & $\%$ \\
Masculino & 37 & 31,4 & 5 & 4,2 & 118 & 100,0 \\
Femenino & 73 & 41,7 & 18 & 10,3 & 174 & 100,0 \\
\hline & Ansiedad POST & \multicolumn{2}{l}{ Depresión POST } & Total POST \\
\hline \multirow{2}{*}{ Fr } & $\%$ & Fr & $\%$ & Fr & $\%$ \\
Masculino & 48 & 34,0 & 9 & 6,4 & 141 & 100,0 \\
Femenino & 110 & 54,2 & 25 & 12,3 & 203 & 100,0 \\
\hline
\end{tabular}

$\mathrm{Fr}=$ Frecuencia de casos \%=Porcentaje de casos

Tabla 8

Comparación por género entre Ansiedad PRE, Depresión PRE, Ansiedad POST y Depresión POST

\begin{tabular}{ccccccc}
\hline Género & \multicolumn{7}{c}{ Rango promedio } \\
& N Pre & Ans & Dep & N Post & Ans & Dep \\
Masculino & 118 & 137,45 & 139,56 & 141 & 146,78 & 156,98 \\
& & & & & & \\
Femenino & 175 & 153,44 & 152,02 & 203 & 190,37 & 183,28 \\
Total & 293 & & & 344 & & \\
\hline
\end{tabular}

$\mathrm{N}$ Pre $=\mathrm{N}^{\circ}$ de casos PRE $\mathrm{N}$ Post $=\mathrm{N}^{\circ}$ de casos POST 
Tabla 9

Estadisticos de contraste por género entre Ansiedad PRE, Depresión PRE, Ansiedad POST y Depresión POST

Prueba U de Mann-Whitney

\begin{tabular}{ccccc}
\hline & $\begin{array}{c}\text { Ansiedad } \\
\text { PRE }\end{array}$ & Depresión PRE & $\begin{array}{c}\text { Ansiedad } \\
\text { POST }\end{array}$ & $\begin{array}{c}\text { Depresión } \\
\text { POST }\end{array}$ \\
Z & $-1,683$ & $-1,515$ & $-4,302$ & $-2,916$ \\
$\begin{array}{c}\text { Sig. asintót. } \\
\text { (bilateral) }\end{array}$ & 0,092 & 0,130 & 0,000 & 0,004 \\
\hline
\end{tabular}

Respecto a la medición de la variable Apoyo Social, en ambas oportunidades los estudiantes presentaron una óptima percepción de esta variable. Esto se puede observar en la Tabla 10.

Tabla 10

Frecuencias y Porcentajes Apoyo Social

\begin{tabular}{clccc}
\hline Categorías & \multicolumn{2}{l}{ Apoyo Social PRE } & \multicolumn{2}{l}{ Apoyo Social POST } \\
\hline Br & $\%$ & Fr & $\%$ \\
Bajo & 23 & 7,8 & 17 & 5,0 \\
Normal & 270 & 92,2 & 322 & 95,0 \\
Total Válidos & 293 & 100,0 & 339 & 100,0 \\
\hline
\end{tabular}

$\mathrm{Fr}=$ Frecuencia de casos \%=Porcentaje de casos

Por último, se encontró una correlación directa, moderada y significativa entre las variables ansiedad y depresión, tanto en la muestra PRE (Rho de Spearman=0,631; $\mathrm{p}=0,000$ ) como en la muestra POST (Rho de Spearman $=0,630 ; p=0,000$ ). Sin embargo, al controlar la variable apoyo social, esta asociación disminuyó ( $\mathrm{PRE}=0,575$; $\mathrm{POST}=0,553)$. Además, la correlación parcial entre depresión y apoyo social, restando la influencia de la ansiedad, es inversa, leve y significativa $\left(\mathrm{r}_{\mathrm{AB} . \mathrm{C}}\right)=-0,319, \mathrm{p}=0,000$ en la muestra PRE y $-0,318, \mathrm{p}=0,000$ en la muestra POST). 


\section{Discusión}

En primer lugar cabe señalar que, debido a que la muestra pertenece a una Facultad específica de la Universidad de Chile, se vuelve difícil generalizar los resultados a otras facultades y/o universidades, en las cuales los estudiantes pueden estar viviendo realidades diversas desde un punto de vista social, económico o académico.

El principal hallazgo de este estudio es que los estudiantes de Odontología de la Universidad de Chile manifiestan sentirse considerablemente más ansiosos que deprimidos. Lo anterior coincide con un estudio chileno más reciente (Alvial et al., 2007), en el cual los estudiantes obtuvieron una mayor prevalencia para el síndrome ansioso que para el depresivo.

En el segundo momento de evaluación ocurre un notorio aumento en el nivel de sintomatología ansiosa, que puede ser explicado por la alta carga académica experimentada por los alumnos en dicha oportunidad, ya que se encontraban en pleno período de pruebas. Este resultado apoya los planteamientos de diversos autores, quienes afirman que las presiones académicas propias de la vida universitaria constituyen una importante fuente de estrés y ansiedad para los estudiantes, actuando en desmedro de su salud mental (Bagés et al., 2008, Degenhardt, Johnson, Klose \& Peterson, 2007). No obstante, no sucede lo mismo para la sintomatología depresiva, la cual permanece sin diferencias significativas entre la primera y segunda medición.

Las notorias diferencias observadas entre las proporciones de casos de ansiedad y casos de depresión pueden deberse a que los estudiantes hayan reportado sentimientos transitorios de ansiedad, como respuesta a situaciones particulares percibidas como amenazantes. Pese a que muchos estudiantes pueden experimentar una alta ansiedad, la mayoría de ellos no alcanzarían a estados emocionales más complicados, como por ejemplo, estados depresivos. Esto podría relacionarse con que la depresión se encuentra asociada a diversos factores genéticos y psicosociales (Arrivillaga et al., 2004) que sobrepasan el contexto propio de la universidad, por lo cual 
para que una persona desarrolle un cuadro depresivo se deberían conjugar experiencias de vida más complejas. Lo anterior se ve respaldado por los hallazgos de Baloglu et al. (2005), quienes encontraron que la depresión correlacionaba en mayor grado con la escala rasgo de ansiedad que con la escala estado de ansiedad de la STAI. Podemos deducir entonces que los estudiantes no presentan un patrón afectivo persistente, donde la causa de la emoción es vaga o indefinida, sino que el cuadro ansioso se manifiesta en relación a una situación específica, lo que correspondería a lo que se conoce como "estado" de ansiedad.

Según Haller, Puskar \& Sereika (2003), altos índices de ansiedad pueden tener importantes consecuencias en el desarrollo de los estudiantes y pueden asociarse a un sustancial deterioro y riesgo de futuros desórdenes psicológicos. Se concluye entonces que, si bien ciertos niveles de ansiedad son necesarios para que los individuos alcancen las metas propuestas y eleven su motivación por ellas, índices elevados de esta podrían generar que los estudiantes pierdan el interés real tanto por la carrera como por sus pacientes, y la motivación inicial que los llevó a elegir dicha carrera y no otra. Con ello, el pasar los ramos y estudiar se volvería una actividad mecánica, perdiendo la satisfacción y el goce por esta.

Los fenómenos descritos anteriormente se presentan en su mayoría en las mujeres de la muestra, reproduciendo los múltiples hallazgos leídos en otras investigaciones, respecto a la mayor presencia de estos cuadros en la población femenina (Azzam et al., 2007; Bates et al., 2000; Degenhardt et al., 2007; González et al., 2004; Guavita \& Sanabria, 2006; Haller et al., 2003; Crawford, Crombie, Henry \& Taylor, 2001). No obstante, lo que resulta más llamativo es que tan solo en el segundo momento existen diferencias realmente significativas entre ambos géneros. Esto va en la línea de lo expuesto en estudios tanto nacionales como internacionales (Salavert \& Soriano, 1996; Barra, Cerna, Kramm \& Véliz, 2006; Cumsille \& Martínez, 1997) que plantean que las situaciones amenazantes impactan más en el género femenino que en el masculino. Debido a que existen diferencias en la socialización entre hombres y mujeres, al parecer, las mujeres no solo están más dispuestas que los hombres a reportar sus sentimientos depresivos y ansiosos admitiéndolos con más facilidad, 
sino que además pueden ser más propensas a experimentar este tipo de síntomas por causas hormonales (Arrivillaga et al., 2004). Por otra parte, los hombres tienen mayor dificultad para reconocer sus emociones, como por ejemplo, el miedo, y para aceptar que pueden verse sobrepasados en algunos contextos (Salavert et al., 1996).

Es preciso destacar la particular situación hallada en el tercer y cuarto año de esta carrera, debido a que presentaron significativamente mayores casos de ansiedad y de depresión que los otros cursos. Esto puede ser explicado por la alta carga académica vivida por estos alumnos, ya que el tercer año concentra una mayor cantidad de asignaturas que los demás cursos y el cuarto año implica el paso definitivo desde el aprendizaje de ciencias básicas hacia los ramos de especialidad, los cuales introducen nuevas exigencias para los estudiantes como, por ejemplo, la atención de pacientes (Universidad de Chile, s/f). Los alumnos de cuarto año requieren desplegar habilidades que van más allá de lo teórico, tales como la capacidad de tolerar la frustración, la resolución de problemas, el desarrollo de estrategias de afrontamiento, entre otras (Alvial et al., 2007; Bovier et al., 2004; Berthelsen et al., 2008). Además, según Alvial et al. (2007), Lange et al. (2006) y Bagés et al. (2008), uno de los factores que más influyen en desencadenar problemas de salud mental en los estudiantes universitarios es la gran cantidad de aprendizajes que ellos deben adquirir en poco tiempo, lo cual los puede llevar a sentirse sumamente agotados mentalmente. En este sentido, las actuales metodologías de evaluación y su calendarización parecen no ser las más adecuadas en términos de resguardar el bienestar emocional de los estudiantes.

Tomando en consideración el año de estudio cursado, los resultados de la presente investigación dan cuenta de que los planteamientos de algunos autores respecto al potencial patogénico que implica el ingreso a la Universidad (Jerez et al., 1996; Guavita et al., 2006; Degenhardt et al., 2007) no se aplican en esta muestra de estudiantes. Esto último, debido a que el primer año no destaca mayormente en la presencia de ansiedad y depresión, situación que se mantiene hasta segundo año. El quinto año tampoco sobresale por sus puntajes en ansiedad y depresión, lo cual no coincide con los resultados de Kamali et al. (2002), quienes al utilizar la 
misma escala en estudiantes de Odontología del Reino Unido encontraron en el quinto año de Odontología los puntajes más altos en la subescala de ansiedad, en comparación con otros cursos.

Debido a la influencia que ejercen los factores ambientales-sociales en la personalidad, asegurar un entorno que otorgue un buen nivel de calidad de vida a estos jóvenes, es vital para apoyar un adecuado desarrollo de su personalidad. En este último punto resulta importante el rol que juega la percepción de apoyo social en la salud mental de las personas. Los estudiantes evaluados sienten, en general, una óptima percepción de apoyo social en ambos momentos. Esto podría estar revelando que, si bien se sienten presionados por la Universidad, cuentan eventualmente con el apoyo de amigos y el de sus familias. Por otro lado, lo anterior estaría relacionado con que la percepción de apoyo social podría verse afectada en gran parte por el estado anímico del sujeto (Barra et al. 2006). Es comprensible entonces que los alumnos reporten bajos niveles de depresión, junto con un apoyo social satisfactorio. Así, una persona aislada y deprimida tenderá a percibir menor apoyo social que una persona que no posea tales sentimientos. El postulado del efecto moderador del apoyo social cobra relevancia, pues ante circunstancias percibidas como amenazantes, la suficiencia de apoyo amortigua la aparición de síntomas de tipo depresivo (García, Martínez \& Maya, 2001).

Se puede concluir que el instrumento que se ha utilizado para medir ansiedad y depresión constituye una herramienta válida y confiable. El análisis realizado demostró que, al igual que hallazgos internacionales que han estudiado sus propiedades psicométricas (Arostegui et al., 2003; Blanch et al., 2003; Dahl, Mykletun \& Stordal, 2001; Baloglu et al., 2005), tanto los ítems de ansiedad como los de depresión se agruparon claramente en torno a dos factores distintos. Esto último sugiere que la ansiedad y la depresión a pesar de estar relacionados, son constructos diferentes.

Para finalizar, se desea señalar que sería de utilidad en este contexto implementar talleres preventivos de autocuidado, dirigidos especialmente a los alumnos de tercer y cuarto año en aquellos momentos del semestre en que se ven expuestos a mayores presiones académicas. Esto, 
junto con una redistribución de las exigencias curriculares, puede contribuir a que la Universidad de Chile vaya logrando una nueva mirada de la educación, que no solo enfatice la excelencia académica, sino también la salud mental.

\section{Referencias}

Acosta, M., Díaz, R. \& Lartigue, T. (2001). Síndrome de Burnout. Desgaste emocional en cirujanos dentistas. Revista ADM, 8(2), 63-77.

Almonte, C., Correa, A. \& Montt, M. (2003). Psicopatología infantil y de la adolescencia (pp. 37-61). Santiago: Mediterráneo Ltda.

Alvial, W., Aro, M., Bonifetti, M., Cova, F., Hernández, M. \& Rodríguez, C. (2007). Problemas de salud mental en estudiantes de la Universidad de Concepción. Terapia Psicológica, 25(2), 105-112.

Arostegui, I., Bilbao, A., Esteban, C., Quintana, J.M., Padierna, A. \& Ruiz, I. (2003). Evaluation of the psychometric characteristics of the Spanish version of the Hospital Anxiety and Depression Scale. Acta Psychiatr Scand, 107, 216-221.

Arrivillaga, M., Cortés, C., Goicochea, V. \& Lozano, T. (2004). Caracterización de la Depresión en Jóvenes Universitarios. Universitas psychologica, 2, 17-25.

Azzam, A., Chandavarkar, U. \& Mathews, C. (2007). Anxiety symptoms and perceived performance in medical students. Research Article Depression and Anxiety, 24,103-111.

Bagés, N., Chacón-Puignau, G., De Pablo, J., Feldman, L., Goncalves, L. \& Zaragoza, J. (2008). Relaciones entre estrés académico, apoyo social, salud mental y rendimiento académico en estudiantes universitarios venezolanos. Universitas psychologica, 7(3), 739-751.

Baloglu, M., Karagozoglu, C. \& Masten, G. (2005). Evidence for differentiating between anxiety and depression in Turkish college students. Social Behavior and personality, 33(6), 579-586.

Barra, E., Cerna, R., Kramm, D. \& Véliz, V. (2006). Problemas de salud, estrés, afrontamiento, depresión y apoyo social en adolescentes. Terapia Psicológica, 24 (001), 55-61.

Barrón, A. \& Sánchez, E. (2001). Estructura social, apoyo social y salud mental. Psicothema, 13(1), 17-23. 
Bascarán, M.T., Bobes, J., Bousoño, M., García-Portilla, M., Parellada, M. \& Sáiz, P. (2008). Escala hospitalaria de Ansiedad y Depresión HADS. Banco de instrumentos básicos para la práctica de la psiquiatría clínica $\left(5^{\circ} \mathrm{Ed}\right.$.). Barcelona: Ars Médica.

Bates, L., Dorian, M., Greig, H., Pickard, M. \& Saint, D. (2000). Alcohol and drug use in second-year medical students at the University of Leeds. Blackwell Science Ltd. Medical education, 34, 148-150.

Bayona, M., Caycedo, C., Forero, C., Montealegre, M., Nieto, C., Novoa, M., et al. (2005). Relación entre perfil psicológico, calidad de vida y estrés asistencial en personal de enfermería. Pontificia Universidad Javeriana. Univ. Psycholog. Bogotá (Colombia) 4(1), 63-75.

Bella, T.T., Odukogbe, A.A., Olayemi, O. Omigbodun, A.O., Omigbodun, O.O. \& Yusuf, O.B., (2006). Stressors and psychological symptoms in students of medicine and allied health professions in Nigeria. Soc Psychiatry Psychiatr Epidemiol, 41, 415-421.

Bellón, J.A., Delgado, A., Lardelli, P. \& Luna, J. (1996). Validez y fiabilidad del cuestionario de apoyo social funcional Duke-UNC-11. Atención Primaria, 18(4), 153-156.

Blanch, J., Bulbena, A., De Pablo, J., Herrero, M. J., Peri, J. M. \& Pintor, L. (2003). A validation study of the hospital anxiety and depression scale (HADS) in a spanish population. Elsevier Inc, 67(6), 361-370.

Bovier, P., Chamot, E. \& Perneger, T. (2004). Perceived stress, internal resources, and social support as determinants of mental health among young adults. Quality of Life Research, 13, 161-170.

Broadhead, W.E., De Gruy, F.V., Gehlbach, S.H. \& Kaplan, B.H. (1988). The Duke- UNC Functional Social Support Questionnaire: Measurement of Social Support in Family Medicine Patients: Medical Care, 26(7), 709-723.

Carrasco, E. \& Osses, S. (2005). Estudio del perfil valórico. El caso de los estudiantes de primer nivel de la carrera de Obstetricia y Puericultura de la Facultad de Medicina de la Universidad de la Frontera. Estudios pedagógicos, 21(1), 7-32.

Carrillo, I. \& Castro, A. (s/f). Convergencias entre ansiedad y depresión en universitarios. Reporte de Investigación. Comité Universitario para la Salud en la UASJ, 35, 1-8. Comité Universitario de Salud Universitaria. Ciudad Juárez, México: Universidad Autónoma de Ciudad Juárez, Programa de Psicología.

Cornejo, M. \& Lucero, M. (2005). Preocupaciones vitales en estudiantes universitarios relacionados con bienestar psicológico y modalidades de afrontamiento. Fundamentos en Humanidades, 2(12), 143-153. 
Crawford, J.R., Crombie, C., Henry, J.D. \& Taylor, E.P. (2001). Brief Report. Normative data for the HADS from a large non-clinical sample. British Journal of Clinical Psychology, 40, 429-434.

Cumsille, P. \& Martínez, M. (1997). Síntomas de depresión en estudiantes de enseñanza media de Santiago. Rev. Chil. Pediatr. 68(2). 74-77.

Dahl, A., Mykletun, A. \& Stordal, E. (2001). Hospital Anxiety and Depression (HAD) Scale: factor structure, item analyses and internal consistency in a large population. British Journal of Psychiatry, 179, 540-544.

Degenhardt, B., Johnson, J., Klose, C. \& Peterson, D. (2007). Depression, anxiety, and perceived hassles among entering medical students. Psychology, Health \& Medicine, 12, 31-39.

Dossi, A., Isper, O., Presta, A., Saliba, C. \& Saliba O. (2006). El estrés en la práctica odontológica. Revista $A D M, 13(5)$, 185-188.

García, M., Martínez, M.F., \& Maya, I. (2001) El efecto amortiguador del apoyo social sobre la depresión en un colectivo de inmigrantes. Psicothema, 13(4), 605-610.

González, J., Granadillo, D., Lara, C. \& Lugo, M. (2004). Depresión, ansiedad y estrés en estudiantes de medicina del área básica y clínica, su relación con el índice de lateralización hemisférica cerebral y el rendimiento académico. Archivos venezolanos de psiquiatría y neurología, 50(103), 21-26.

González, M. \& Landero, R. (2006). Variables asociadas a la depresión: un modelo de regresión logística. Revista Electrónica de Metodología Aplicada, 11, 16-30.

Guavita, P. \& Sanabria, P. (2006). Prevalencia de Sintomatología Depresiva en una población estudiantil de la Facultad de Medicina de la Universidad Militar Nueva Granada. Rev. Fac. Med. Univ. Nac . Colomb., 54(2), 76-85.

Haller, L., Puskar, K. \& Sereika, S. (2003). Anxiety, somatic complaints, and depressive symptoms in rural adolescents. Journal of Child and Adolescent Pschyatric Nursing, 16, 102-111.

Jerez, M., Rioseco, P., Valdivia, S., Vicente, B. \& Vielma, M. (1996). Nivel de salud mental en los estudiantes de la Universidad de Concepción. Revista de Psiquiatría, 13(3), 158-165.

Kamali, F., Lowry, R.J. \& Newbury-Birch, D. (2002). The changing patterns of drinking, illicit drug use, stress, anxiety and depression in dental students in a UK dental school: a longitudinal study. British Dental Journal, 192, 646-649. 
Kitamura, T. \& Matsudaira, T. (2006). Personality Traits as Risk Factors of Depression and Anxiety Among Japanese Students. Journal of Clinical Psychology, 62(2), 97-109.

Lange, I. \& Vio, F. (2006). Guía para Universidades Saludables y otras Instituciones de Educación Superior. Santiago. Elaboración conjunta del Instituto de Nutrición y Tecnología de los Alimentos y la Pontificia Universidad Católica.

Reynaldos, C. \& Saavedra, E. (2006). Caracterización cognitiva y emocional de los estudiantes de la Universidad Católica del Maule: Años 1999, 2001, 2003. Estud. pedagóg. 32(2), 87-102.

Salavert, L. \& Soriano, J. (1996). The Hospital Anxiety and Depression Scale questionnaire in physical danger and evaluation. Counselling Psychology Quartely, 9, 281-296.

Snaith, R. P. (1992). Availability of the Hospital Anxiety and Depression (HAD) Scale. Br J. Psychiatry, 161, 422.

Snaith, R. P. \& Zigmond, A. S. (1983). The Hospital Anxiety and Depression Scale. Acta Psychiatrica Scandinavica, 67, 361-370.

Universidad de Chile (2006). Reglamento de Estudios de Odontología conducente a la obtención del Grado de Licenciado en Odontología y del Titulo Profesional de Cirujano Dentista. Recuperado el 25 de mayo de 2009 desde http://odontologia.uchile.cl/?_nfpb=true\&_pageLa bel=conUrlOdontologia\&url=51649

Universidad de Chile (s/f). Plan de estudios de la carrera de Odontología. Recuperado el 25 de mayo de 2009 desde http://odontologia.uchile. $\mathrm{cl}$ ??_nfpb=true\&_pageLabel=conUrlOdontologia\&url=50884

Fecha de recepción: 01 de agosto de 2011.

Fecha de aceptación: 20 de diciembre de 2011. 